\title{
Precise radiolarian age constraints on the timing of ophiolite generation and sedimentation in the Dazhuqu terrane, Yarlung-Tsangpo suture zone, Tibet
}

\author{
SERGEY V. ZIABREV', JONATHAN C. AITCHISON', \\ ALEXANDRA V. ABRAJEVITCH ${ }^{1}$, BADENGZHU², AILEEN M. DAVIS' \& HUI LUO ${ }^{1,3}$ \\ 'Tibet Research Group, Department of Earth Sciences, University of Hong Kong, Pokfulam Road, Hong Kong SAR, P.R. \\ China (e-mail: email: jona@hku.hk) \\ ${ }^{2}$ Geological Team \# 2, Tibet Geological Survey, Lhasa, Tibet, P.R. China \\ ${ }^{3}$ Present address: Nanjing Institute of Geology and Palaeontology, Laboratory of Palueobiology \& Stratigraphy, Academia \\ Sinica, Nanjing 210008, P.R. China
}

\begin{abstract}
Well-preserved, abundant radiolarians provide high-precision biostratigraphic age constraints on the timing of the eruption of ophiolitic basalts exposed along the Yarlung-Tsangpo suture zone in southern Tibet. Dazhuqu terrane ophiolites were generated in an intra-oceanic supra-subduction zone setting within a relatively short $(<10 \mathrm{Ma})$ interval from late Barremian to late Aptian. Accumulation of sediments upon the newly generated ophiolite initially occurred in a series of discrete rift-controlled sub-basins associated with various spreading centres. An increasing flux of are-derived volcaniclastic sediment up-section indicates ncarby volcanic arc activity. The Dazhuqu terrane developed in an intra-oceanic setting within Tethys where it was isolated from any continental influcnce.
\end{abstract}

Keywords: Radiolaria, Yarlung-Tsangpo suture, Tibet, ophiolites, biostratigraphy.

The Yarlung-Tsangpo suture zone in Tibet marks the tectonic boundary between Eurasia and India. The previously vast Tethys ocean finally closed along the suture during their Cenozoic collision. Most of this occan was lost through subduction during convergence or was overridden during collision. All that remains now occurs within a suture zone of a few kilometres width. A belt of ophiolitic bodies is the most traceable feature along the Yarlung-Tsangpo suture zone, in south Tibet and beyond. Several ophiolitic massifs form a nearly continuous exposure that stretches cast-west over a distance of c. $150 \mathrm{~km}$ near Xigaze, $250 \mathrm{~km} \mathrm{SW}$ of Lhasa. These rocks are assigned to the Dazhuqu terrane and interpreted as having originated in an intra-oceanic supra-subduction zone setting (Aitchison et al. 2000, 2002a). This interpretation of the origin of the Dazhuqu ophiolite is supported by detailed mineralogical and geochemical studies in the Xigaze area (Hébert et al. 2000; 2001). The presence of these rocks together with other intra-oceanic terrancs along the suture led to the suggestion of Aitchison et al. (2000) that a southfacing intra-oceanic subduction system once lay within the Tethys.

Knowledge of the timing of generation of the Dazhuqu ophiolite is a significant issue for understanding regional geology and has implications for development of models for Tethys evolution. Marine sedimentary cover on mafic volcanic rocks was previously dated as late Albian to possibly early Cenomanian (Marcoux et al. 1982) or early Cenomanian (Wu 1986) on the basis of radiolarians reported from the Xigaze district. These ages appeared to be some $20 \mathrm{Ma}$ younger than a radiometric age of $120 \pm 10 \mathrm{Ma}$ inferred from $\mathrm{U}-\mathrm{Pb}$ whole-rock analyses of gabbros, dolerites and basaltic lavas reported from the same area (Göpel et al. 1984). Until now, no further biostratigraphic study of the Dazhuqu terrane has been published. Meanwhile, Cretaceous radiolarian biostratigraphy for the Tethyan regions has developed significantly (Jud 1994; O’Dogherty 1994; Baumgartner et al. 1995). This opens the possibility for obtaining more accurate biostratigraphic information that will permit better recognition of any sequence of events related to development of the ophiolite. In the course of our study of the Dazhuqu terrane, we obtained new data on the lithostratigraphy and radiolarian biostratigraphy of the sedimentary succession overlying the ophiolite. These results constrain the timing of ophiolite formation and permit a better understanding of the development of sedimentary basins on this newly gencrated supra-subduction zone crust.

\section{Regional tectonic framework}

Six tectonostratigraphic units (terranes) that developed before India-Eurasia collision are rccognized (Aitchison et al. 2000) within and bounding the Yarlung-Tsangpo suture zone (Fig. 1). The northern side of the suture is delineated by the Lhasa terrane, a microcontinental block that had detached from the northern periphery of Gondwana and docked with Asia by the Late Jurassic (Allègre et al. 1984; Yin \& Harrison 2000).

A $5000-8000 \mathrm{~m}$ succession of volcaniclastic turbidites (Xigaze terrane) lics to the south of the Lhasa terrane. The Xigaze terrane is thrust northwards over upper Oligocene-lower Miocene Gangrinboche facies conglomerates (Aitchison et al. 2002b). At its southern boundary the terrane lies in the footwall of another north-directed thrust with Dazhuqu terranc ophiolitic rocks or Palcogene Liuqu Conglomerate (Davis ef al. 2002) in the hanging wall. Rare fossils indicate that the turbidites have a late Albian to Coniacian stratigraphic range (Wiedmann \& Dür 1995; Wan et al. 1998). As the oldest known fossils are not from the base of the section it is possible that sedimentation may have commenced before the late Albian. The top of the Xigaze turbidite sequence is truncated by crosion. Xigaze terrane rocks are interpreted as a forearc succession that developed in association with north-directed subduction beneath the Lhasa tcrrane (Shackleton 1981; Burg \& Chen 1984; Girardeau et al. 1984; 

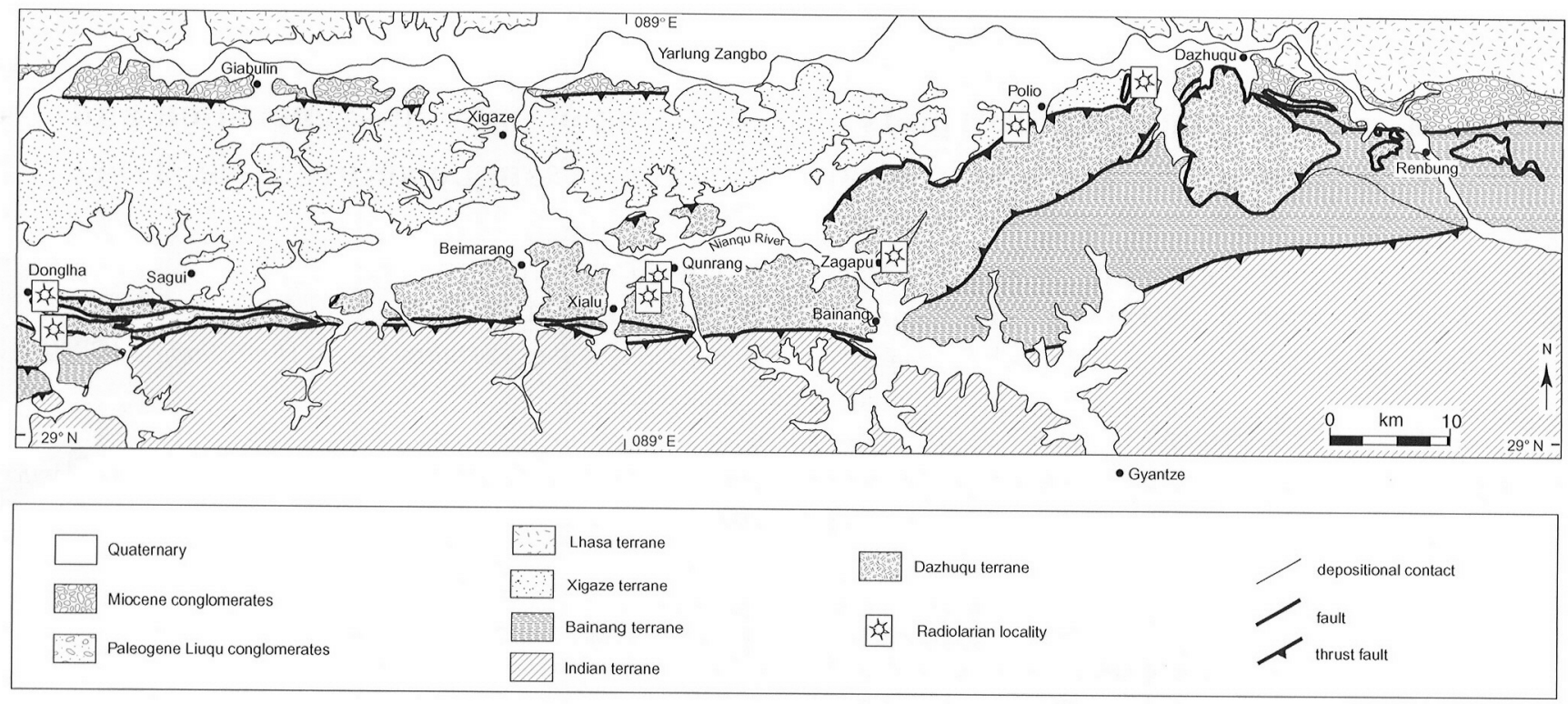

Fig. 1. Simplified geological map showing distribution of Dazhuqu terrane rocks near Xigaze (modified from Wang et al. 1987) and localities of the sections studied.

Einscle et al. 1994; Dürr 1996). Development of the terrane was related to evolution of the magmatic are along the southern edge of the Lhasa terrane (Einscle et al. 1994; Dürr 1996). Although the Xigaze terrane is conventionally regarded as being floored by the Dazhuqu ophiolite (e.g. Burg \& Chen 1984; Girardcau et al. 1984; Einsele et al. 1994; Dürr 1996), these two units are ubiquitously in tectonic contact and have recently been interpreted as separatc terranes (Aitchison et al. 2000, 2002a).

A tectonic sliver of intra-oceanic island arc rocks (Zedong terrane) crops out between Lhasa and Dazhuqu terranes near Zedong and Luobusa. This terrane is bounded by north-directed thrusts related to the Renbu-Zedong thrust system of Yin et al. (1994). The basal contact of the section lies at a fault and begins with a thin (several metres) succession of arc tholeiitic lavas overlain by a c. $15 \mathrm{~m}$ sequence of red ribbon-bedded chert followed by $1000 \mathrm{~m}$ or more of volcaniclastic breccias. The succession is cut by numerous basaltic-andesite dykes and minor intrusions of diorite and leucogranitc (McDermid et al. $200 \mathrm{la}$ ). Both radiometric and biostratigraphic data indicate the onset of magmatism in late Mid-Jurassic time. Radiometric ages determined by $\mathrm{U} / \mathrm{Pb}$ ion microprobe zircon analysis together with ${ }^{40} \mathrm{Ar}{ }^{39} \mathrm{Ar}$ ages for hornblende (McDermid et al. 2001b, 2002) arc in accord with radiolarian faunas of Bajocian-early Callovian age in the underlying chert. The terrane is interpreted as remnants of intra-oceanic magmatic are (Aitchison et al. 2000, $2002 \mathrm{a}$; McDermid et al. 2001a) similar to other terranes known from elsewhere along the suture in NW India and Pakistan.

The Dazhuqu terrane consists of a series of ophiolitic bodies traceable along the Yarlung-Tsangpo suture zone with a major zone of outcrops near Xigaze (Aitchison et al. 2003). In this area the ophiolite is thrust northwards over the Xigaze terrane (Burg 1983; Wang et al. 1987). The southern margin of the terrane is defined, in many arcas, by the Miocene north-directed RenbuZedong thrust (Yin et al. 1994, 1999), which places Indian Plate rocks over the ophiolite. In the Bainang district, where there is a sigmoidal bend in the Yarlung-Tsangpo suture zone, earlier contacts with the Bainang terrane can be observed at southdirected thrusts that are in places truncated by strikc-slip faults (Girardeau et al. 1985a; Ratschbacher et al. 1994).
Several ophiolitic massifs in the Xigaze area form a nearly continuous belt over $175 \mathrm{~km}$ long and up to $25 \mathrm{~km}$ wide. Ophiolitic sections are mostly north-side up with the sequence repeated across dextral strikc-slip faults. Although tectonically disrupted and heavily attenuated, sections locally display a complete ophiolitic sequence from fresh $\mathrm{Cr}$ diopside-rich harzburgites to marinc sedimentary cover on mafic volcanic rocks (Nicolas et al. 1981; Girardeau et al. 1984, 1985a, 1985b). Aitchison ef al. (2000, 2002a) interpreted the Dazhuqu terrane ophiolite as having originated in an intra-oceanic supra-subduction zone setting and this is supported by detailed mineralogical and geochemical studies in the Xigaze area (Hébert et al. 2000, 2001 ). Palaeomagnetic study of the sedimentary succession overlying mafic rocks of the Dazhucu terrane indicates its deposition in an cquatorial area, $1000-1500 \mathrm{~km}$ south of Asia's margin (Abrajevitch et al. 2001).

The Bainang terrane, on the southern side of the suture zone, was recently discriminated by Aitchison et al. (2000), who interpreted it as a subduction complex. The terrane lies between ophiolitic rocks of the Dazhuqu terrane to the north and the Indian terrane to the south. It contains units previously referred to as infra-ophiolitic thrust sheets of radiolarites (Burg \& Chen 1984) or Upper Jurassic to Lower Cretaceous red radiolarites (Girardcau et al. 1984). Good exposures exist near Donglha, Xialu and Bainang. In most sections studied the terrane is chert dominated and is characterized by an overall south-younging tectonic pile of oceanic lithologies in which north-younging successions are stacked by a series of south-verging thrusts. Radiolarians reported from siliceous rocks near Xialu range in age from Mid-Jurassic to Early Cretaceous (Aptian) (Wu 1993; Matsuoka et al. 2001, 2002). Our detailed geological mapping and investigations of radiolarian biostratigraphy elucidate the structure, stratigraphy and evolution of the terrane (Ziabrev et al. 2000; Ziabrev 2002). Radiolarians allow reconstruction of a relict stratigraphy that records a long history of sedimentation in different portions of Tethys since the Late Triassic. Stratigraphy within the terrane records the northward travel of an oceanic plate and its approach towards a south-facing intra-oceanic subduction zone where accretion occurred from late Aptian to at 
least the Campanian (Ziabrev 2002). Variations in structural style across the terrane indicate deformation at different depths and vertical growth of the wedge dominant over lateral accretion. Tectonostratigraphic features specific to the Bainang terrane reffect its development in a remote intra-oceanic setting (Ziabrev 2002).

Passive margin rocks of the Indian tcrrane or Tethyan (Tibetan) Himalaya lie south of the suture. Thick Permian to Paleogene continental rise deposits (Liu \& Einsele 1994) merge southward into a continuous Ordovician to Eocene shelf sedimentary succession of marine carbonates, sandstone, siltstone and shale (Bureau of Geology and Mineral Resources of Xizang Autonomous Region 1993; Jadoul et al. 1998). Disruption of northern Indian margin rocks into widespread regional mélange zones accompanied Paleogene collision between an intra-oceanic island are and the northern margin of India (Liu et al. 2000; Liu \& Aitchison 2002). Passive margin sedimentation finally ceased with the Cenozoic India-Asia collision.

The original disposition of terranes within the suture zone has been greatly disrupted and former relations between terranes are not well constrained, making reconstruction of the tectonic evolution of the area difficult. Most early models (Allègre et al. 1984; Searle et al. 1987) invoked the existence of a single Andean-type convergent plate margin along the northern side of Tethys, although the possibility of additional subduction zones was considered by some workers (Proust et al. 1984). The cooccurrence and north-south distribution of the Zedong (magmatic arc), Dazhuqu (forearc ophiolite) and Bainang (subduction complex) terranes led to their interpretation as evidence for a south-facing intra-oceanic subduction system that lay within the Tethys (Aitchison et al. 2000), and the existence of more than one convergent margin. Analogy with the modern western Pacific and SE Asia suggests that reality may have been even more complex. As more details and constraints on the evolution of terranes within the Yarlung-Tsangpo suture zone become available the complexity and sophistication of models for this zone is likely to increase.

\section{Previous work}

Most of the marine siliccous and fine-grained clastic deposits that cover the ophiolite crop out along the northern margin of the Dazhuqu terranc. These deposits are referred to as the Chongdu Formation (Cao (1981) citcd by Bureau of Geology and Mincral Resources of Xizang Autonomous Region 1993). The first lithoand biostratigraphic information became available in reports of Sino-French expeditions to this area in the early 1980s (Marcoux et al. 1982; Girardeau et al. 1984, 1985a, 1985b). Previously the cherts as well as fine-grained clastic deposits have been accorded late Albian and possibly early Cenomanian ages based on radiolarians (Marcoux et al. 1982). Other radiolarian fossils described from these deposits were interpreted as being of late Albian to early Cenomanian age ( $\mathrm{Li} \& \mathrm{Wu}$ 1985) or of early Ccnomanian age (Wu 1986) based on correlation with the Archaeospongoprunum techamaensis Zone (Pessagno 1976) of California. As the precision of Upper Mesozoic radiolarian biostratigraphy has greatly improved since these pioncering studies it is now possible to reassess the biostratigraphy and sedimentary evolution of the Dazhuqu ophiolitic terrane. Radiolarian ages from deposits immediatcly overlying the ophiolite should constrain the timing and duration of the ophiolite generation event.

\section{Methods}

The sedimentary cover of the ophiolite has been sudied at seven sections (Fig. 2) in the Xigaze area, most of which were reported by Sino-French expeditions to this area in the early 1980s (Girardeau es al. 1984). A detailed log was made of each section. Special attention was paid to the nature of contacts between pillow basalt or breccia and overlapping deposits, to recognize any tectonic disruption. Seventy-five samples were collected for micropalacontological investigation and treated in dilute IJF $\pm \mathrm{HCl}$ to extract radiolarians. Species identification and age assignments are chiefly based on recent taxonomic studies and biostratigraphic zonation of Mid-Cretaceous Tethyan radiolarians (Jud 1994; O'Dogherty 1994). The first and last occurrences of some species are derived from an unpublished composite range chart of $\mathrm{O}^{\prime}$ Dogherty snl Jud ( $\mathrm{O}^{\prime} \mathrm{D}$ ogherty \& Guex, pers. comm.). These range charts utilize the Unitary Association (UA) method (Guex 1991), and numerical UA ages calibrated to the Gradstein et al. (1994) time scale are applied herein. Over 50 radiolarianbased ages were thus acquired in the course of this study. To lest the appropriateness of the western Tethys Mid-Cretaceous radiolarian range chart (O'Dogherty 1994) to our far eastern Tethyan study area as many taxa as possible were identified from each sample. The absence of unexpected co-occurrences indicates no detectable diachroneity in the distribution of taxa between western and eastern Tethys regions, suggesting that the range chart of O'Dogherty (1994) is applicable all along the Tethys.

\section{Stratigraphy}

Sedimentary sections exposed along $175 \mathrm{~km}$ strike length of the ophiolite were examined in detail. The sections are cither situated along the northern boundary of the Dazhuqu terrane (Donglha-1, Polio and Dazhuqu) or tectonically interleaved within the ophiolite (Donglha-2, Qunrang and Zagapu) (Fig. 2). Various marine sedimentary lithologies overlie the ophiolite and include chert, siliceous mudstone and finegrained volcaniclastic rocks. Exposure is near-continuous, with the uppermost levels of sections truncated by faults. Minor normal faulting within sections has locally eliminated small portions of some sections. Undisturbed depositional contacts with underlying pillow lavas or pillow breccias were recognized in most sections, although some minor shearing locally occurs near the contact. Sections adjacent to the Xigaze terrane are everywhere in fault contact with turbiditic rocks of the Xigaze (jtoup.

Near the village of Donglha, $50 \mathrm{~km}$ SW of Xigaze a well-cxposed scction (Donglha-1, 29 $08.392^{\prime} \mathrm{N}, 088^{\circ} 24.575^{\prime} \mathrm{F}$ ) crops out on a hill slope beside an irrigation canal. Putplish red bedded $(412 \mathrm{~cm})$ chent is the dominant lithology although some intercalated $(2 \cdots 5 \mathrm{~cm})$ chent and siliceous mudstone occurs at the top of the section. Sparse tuflaceous laminae and thin $(0.5-4 \mathrm{~cm})$ layers of felsic tuff become more abundant up-section. The chert depositionally overlics basaltic pillow breccia, the uppermost $20 \mathrm{~cm}$ of which is encrusted by massive chert. Two thin $(5 \mathrm{~cm})$ chent layers occur within the pillow breccia, $3 \mathrm{~m}$ and $6 \mathrm{~m}$ below the contact. The lower portion ( $14 \mathrm{~m}$ ) of the cliert erops out in a steeply north-dipping to vertical cast-west-1rending succession. Small-scale (lens of centimetres) south-verging intrafolial asymmetric folds indicate southdirected thrusting with a dextral strike-slip component. The upper portion of the sequence is folded and separated from lower parts of the section by $10 \mathrm{~m}$ of non-exposure. Many samples yielded abundant and wellpreserved radiolarians (Table 1; Fig. 3) that allow precisc age determination. The lower chert (samples 2-7) ranges from upper Barremian (UA H. asseni Zone) to lower Aptian (UA5/6 H. verbereki Subzonc) and both the upper and folded cherts (samples 7 20) are upper $A p t i a n$ (UA6/7 to UA8 $T$. costata Subzone). The total range of the Donglha- 1 section ( 6 . $16 \mathrm{~m}$ thick) is upper Barremian to upper Aptian.

A tectonic sliver ( $100 \mathrm{~m}$ thick) of purplish red and greenish grey siliccous mudstones with minor greenish grey chert (Donglha-2, $\left.29^{\circ} 06.954^{\prime} \mathrm{N}, 088^{\circ} 25.518^{\prime} \mathrm{E}\right)$ crops out $4 \mathrm{~km} \mathrm{SL}$ of Dongtha-1. This section is bounded by north-directed thrusts and structure is complicated by folds and shear zones with the possibility of tectonic repetitions, making it difficult to establish the original stratigraphy. Ten samples were collected and most possess similar radiolarian assemblages. Three assemblages with the widest stratigraphic range are used for correlation. 


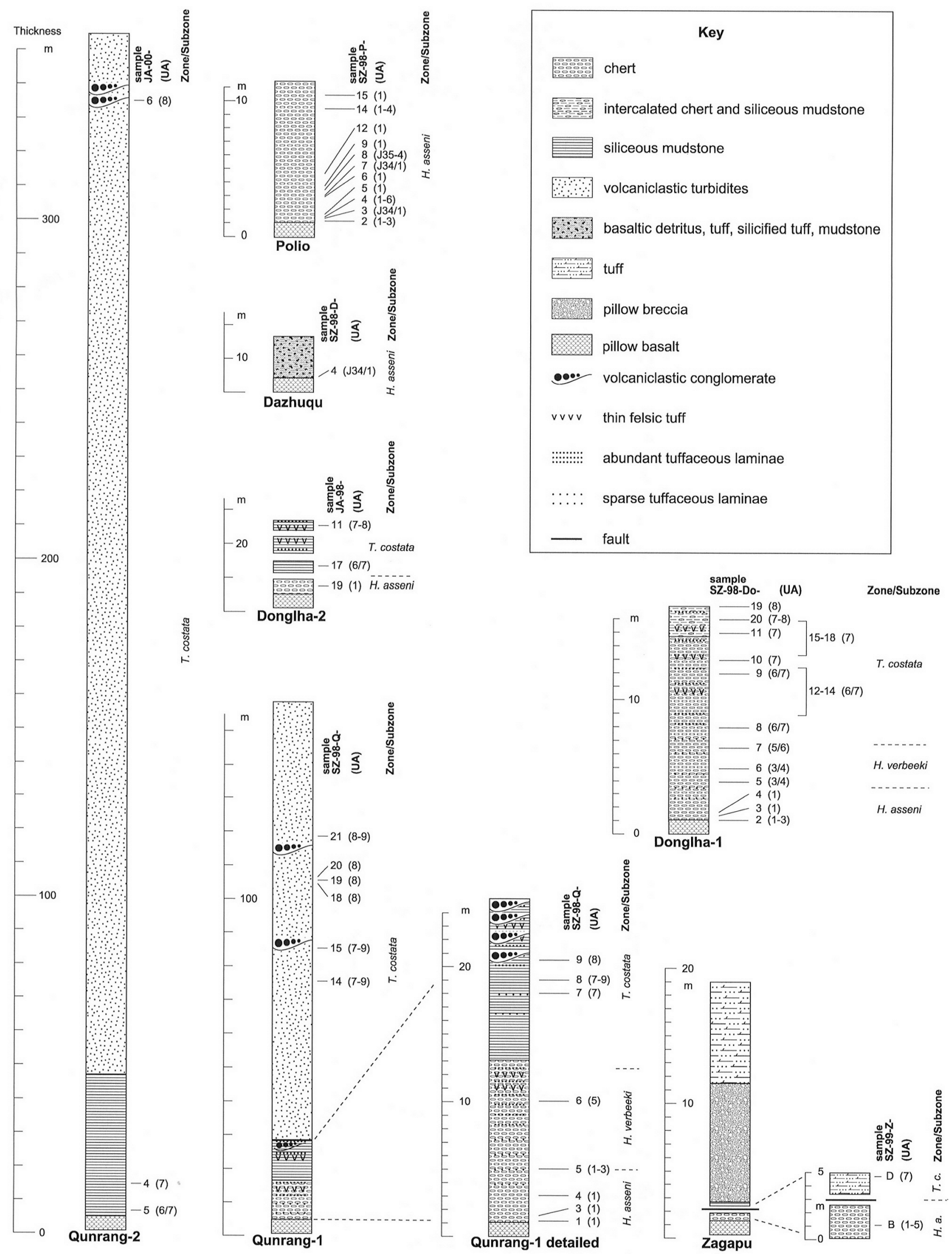

Fig. 2. Lithological columns of the sedimentary sections overlying pillow breccia of the Dazhuqu terrane, with the positions of samples and their correlation with Unitary Associations (UA) and zones or subzones. Details of some sections or their lower portions are shown at a larger scale on the right. 
Table 1. Occurrence of radiolarian species in the Dazhuqu terrane and the ages of radiolarian assemblages with respect to Unitary Associations

\begin{tabular}{|c|c|c|c|c|c|c|c|c|c|c|c|c|c|c|c|c|c|c|c|c|c|c|c|c|c|c|c|c|c|c|c|c|c|}
\hline sample number & & & & & & & $38-5$ & & & & & & & & -98- & & & & & $z-9$ & & & & & & 0. & SZ-9 & & & & sZ-98 & & \\
\hline species list & & & & & & & & & & & & & & & & & & & & & & & & & & & & & & & & & \\
\hline Acaeniotyle diaphorogona Foreman & $\bullet$ & $\cdot$ & $\bullet$ & $\cdot$ & $\cdot \cdot$ & $\bullet$ & $\bullet$ & $\bullet$ & $\cdot$ & & $\bullet$ & & $\bullet \bullet$ & $\cdot$ & & & $\bullet$ & $\cdot \cdot$ & $\bullet$ & & & & & & & - & & & $\bullet$ & - & & 0 & \\
\hline Acaeniotyle umbilicata (Rüst) & $\cdot \bullet$ & $\cdot$ & $\cdot$ & $\bullet$ & & $\bullet$ & $\bullet$ & - & $\bullet$ & & $\bullet$ & & $\bullet \bullet$ & & $\bullet \cdot$ & $\cdot$ & $0^{\circ}$ & $\cdot \bullet$ & $\cdot \cdot$ & & & & $\cdot 1$ & & & $\cdot$ & & & $\cdot$ & & $\bullet$ & & \\
\hline Aurisatumalis carinatus (Foreman) & & & & & & & & & & & & & & & & & $\bullet \cdot$ & & & & & & & & & & & & & & & & \\
\hline Becus gemmatus Wu & $\bullet$ & $\cdot$ & & $\bullet$ & - & $\bullet$ & & & $\cdot$ & & $\cdot \bullet$ & & $\cdot$ & & & & $\cdot$ & 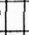 & & & & & & & & $\cdot$ & & & $\cdot$ & 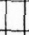 & 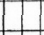 & & \\
\hline Becus helenae (Schaaf) & & & o & & & & & & & & & & & & & & $\bullet$ & 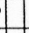 & & & & & & & & & & & $\bullet$ & & $\bullet \bullet$ & $\cdot$ & \\
\hline Becus horridus (Squinabol) & & & & & & - & 0 & - & $\bullet$ & & - $\bullet$ & $\bullet$ & & & & & & & & & & & & & & & & & & & & & \\
\hline Cecrops septemporatus (Parona) & & & & & & & & & & & & & & & & & & & & & & & & & & & & & 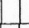 & & & & \\
\hline Crolanium puga (Schaaf) & & & & $\bullet$ & - & $\bullet$ & $\cdot$ & $-\circ$ & $\bullet$ & & $\cdot$ & $\bullet$ & $\bullet \bullet$ & & & & - & $\cdot$ & $\bullet$ & & & & & - & $\cdot$ & - & 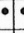 & & $\bullet$ & $\bullet$ & $\bullet$ & 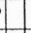 & $\bullet$ \\
\hline Crucella bossoensis Jud & & & & & & & & & & & & & & & & & - & 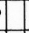 & & & & & & & & & & & $\bullet$ & & & & \\
\hline Crucella euganea (Squinabol) & & & & & - & $\cdot$ & $\cdot 1$ & - & $\cdot$ & & - $\bullet$ & $\bullet$ & 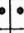 & & & & & & & & & & & & & $\cdot$ & & & & & & & \\
\hline Crucella gavalai O'Dogherty & & & $\cdot \bullet$ & $\bullet$ & $\cdot 1$ & & & -1 & $\cdot$ & & & & & & & & & & & & & & & & & & & & & & & & \\
\hline Crucella hispana O'Dogherty & & & & & & & & & & & & $\bullet$ & $\bullet \cdot \bullet$ & & $\bullet^{\circ}$ & & 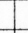 & & 0 & $\bullet$ & & - & & & $\cdot 10$ & $\cdot$ & & & & & & & \\
\hline Cryptamphorella clivosa (Aliev) & $\cdot \bullet$ & $\bullet$ & $\bullet \bullet$ & & $\bullet$ & & & & $\cdot$ & $\cdot$ & $\cdot$ & $\bullet$ & & $\bullet$ & & & -1. & & $\cdot \bullet$ & & & & & & & 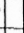 & & 0 & $\circ$ & $\cdot$ & $\bullet$ & & $\bullet$ \\
\hline Cryptamphorella crepida O'Dogherty & $\cdot$ & & $\bullet$ & & & & & & & & & & & & & & & $\cdot$ & & & & & & & & & & & & & & & $\bullet$ \\
\hline Cryptamphorella gilkeyi (Dumitrica) & & & & & & & & & & & & & & & & & & & & & & & & & & & $\bullet$ & & & & & & \\
\hline Cyclastrum infundibuliforme Rüst & & & & & & & & & & & & & & & & & - & of & & & & & & & & & & & & & & & \\
\hline Dactyliodiscus lenticulatus Jud & & $\cdot$ & & & & -・ & $\cdot$ & $\cdot 0$ & & $\cdot$ & - & $\cdot$ & 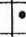 & & & & & $\cdot \bullet$ & & $\cdot$ & & & & & & & & & & & & & \\
\hline Dactyliosphaera maxima (Pessagno) & & & & & & • & $\cdot$ & & & $\cdot$ & $\cdot$ & $\bullet$ & $\cdot \bullet$ & & $\cdot$ & & 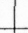 & & & & & & & & $\cdot 0$ & $\cdot$ & & & & & & & \\
\hline Deviatus diamphidius (Foreman) & & & & $\bullet$ & & $\bullet \bullet$ & $\cdot$ & & $\bullet$ & & & 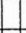 & $\bullet$ & & - $\bullet$ & & $\bullet$ & 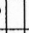 & & & & & & & & & & & $\bullet$ & & & & \\
\hline Dicerosatumalis amissus (Squinabol) & & $\cdot$ & $\cdot$ & $\bullet$ & & $\bullet$ & $\cdot$ & $\cdot$ & & & $-\bullet$ & $\bullet$ & & & & & $\cdot$ & $\cdot$ & & & & & & & & & + & & $\cdot$ & & $\cdot$ & & \\
\hline Dictromitra communis (Squinabol) & $\bullet \bullet$ & $\bullet$ & -) $\bullet$ & $\bullet$ & $\cdot 1$ & - $\bullet$ & $\cdot$ & - & - & - & -1 & $\cdot$ & $\cdot 0$ & & 0 & & $\bullet \cdot$ & & $\cdot \cdot$ & & $\cdot$ & $\bullet$ & $\bullet$ & & $\cdot 10$ & $\cdot$ & - & $\cdot$ & $\cdot$ & & $\cdot \bullet$ & & \\
\hline Dictyomitra excellens (Tan) & & & & $\bullet$ & & & & & & & & & & & & & - & - & & & & & & & & & & & & & & & \\
\hline Godia decora (Li et Wu) & & & & & $\cdot$ & $\bullet$ & $\cdot$ & & $\cdot$ & $\bullet$ & - $\bullet$ & $\cdot$ & $\cdot \cdot$ & & $\bullet \cdot$ & & & & & $\bullet$ & & & & & $\cdot$ & $\cdot$ & & - & & & & & \\
\hline Hexapyramis pantanellii Squinabol & & & & & & & 0 & & & & & & & & - & & & & & & $\cdot$ & & & & & $\bullet$ & & - & 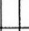 & & & & \\
\hline Hiscocapsa asseni (Tan) & $\bullet$ & & $\bullet$ & $\bullet$ & - & & $\cdot 1$ & & & $\bullet$ & & $\cdot$ & & & & $\cdot$ & $\bullet$ & 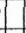 & $\bullet$ & & & & & & & $\cdot$ & & & $\bullet$ & & & & \\
\hline Hiscocapsa grutterinki (Tan) & & & & $\cdot$ & & & $\cdot$ & & & - & & & & & & & & & & & & & & & & $\cdot$ & & & $\bullet$ & & 0 & & \\
\hline Hiscocapsa kaminogoensis (Aita) & & & & & & & & & & & & & & & & & & & & & & & & & & & & & $\bullet$ & & $\cdot$ & & \\
\hline Hiscocapsa orca Foreman & & & & & & & & & & & & & & $\cdot$ & & $\bullet$ & & & & & & & & & & & & & & & $\cdot 1 \cdot$ & & $\cdot$ \\
\hline Hiscocapsa uterculus (Parona) & & & & & & & & & & & & & & & & 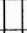 & $\cdot$ & & & & & & & & & & - & & $\cdot$ & & 0 & & \\
\hline Obeliscoites perspicuus (Squinabol) & & $\cdot$ & & & & & - & & & & - & & & & & 0 & & & & & 0 & & & $\bullet$ & & & & & 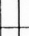 & & 10 & & \\
\hline Obeliscoites vinassai (Squinabol) & & & & & $\bullet$ & & & & & & & & & & & & & & & & & & & & & & & & & & & & \\
\hline Pantanellium lanceola (Parona) & & & & $\bullet$ & & & & & $\cdot$ & & & & & $\bullet$ & & $\bullet$ & $\bullet \bullet$ & $\cdot$ & & & & & & & & & & & $\cdot$ & & $\bullet$ & & \\
\hline Parvicingula boesii (Parona) & & & & & & & & & & & & & & & & $\bullet$ & 0 & & & & & & & & & & $\bullet$ & & $\bullet$ & & $\bullet$ & & - \\
\hline Parvicingula usotanensis Tumanda & & & & & & & & & & & & & & & & 0 & & & & & & & & & & & & & . & $\cdot$ & & & \\
\hline Podobursa typica (Rüst) & & & & & & & & & & & & & & & & & - & - & & & & & & & & & & & $\cdot$ & 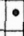 & 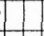 & & \\
\hline Podobursa tythopora (Foreman) & & & & & & & & & & & & & & & & - & $\bullet$ & & & & & & & & & & & & & o & $\bullet$ & & \\
\hline Pseudoaulophacus (?) florealis Jud & $\circ$ & & & & & & & & & & & & & & & $\cdot$ & $\bullet \cdot$ & & & & & & & & & & & & & $\cdot$ & D & & \\
\hline Pseudodictyomitra carpatica (Lozyniak) & $\cdot$ & $\cdot$ & $\bullet$ & & & & & & & & & & & $\circ$ & & $\cdot$ & $\cdot \bullet$ & & $\cdot$ & & & & & & & & o & $\circ$ & & & - $\bullet$ & & \\
\hline Pseudodictyomitra hornatissima (Squinabol) & $\bullet$ & & & $\cdot$ & - & & $\cdot$ & - & $\bullet$ & & $\cdot$ & $\cdot$ & $\bullet \cdot$ & & & 0 & $\cdot$ & & $\bullet$ & $\bullet$ & $0 \cdot$ & $\cdot$ & $\cdot$ & $\cdot \cdot$ & $\cdot \cdot$ & $\cdot$ & & $0^{\circ}$ & 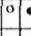 & $\bullet$ & 0 & & \\
\hline Pseudodictyomitra leptoconica (Foreman) & - & & & & & & & & & & & & & & & & $\bullet$ & 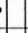 & & & & & & & & & & - & & $\cdot$ & $\bullet \cdot$ & & \\
\hline Pseudodictyomitra lilyae (Tan) & $\bullet$ & & & & & & & & & & & & & & & & $\bullet$ & & & & & & & & & & & & & - & & & \\
\hline Psoudodictyomitra pentacolaensis Pessagno & & & & & & & & & & & & & - & & & & & & & & & & $\cdot \cdot$ & $\cdot \cdot$ & & $\cdot$ & & & & & & & \\
\hline Psoudooucyntis hanni (Tan) & $\bullet \cdot$ & $\cdot$ & & $\cdot$ & & - & - & - & $\bullet$ & $\cdot$ & - & $\bullet$ & $\cdot$ & & - & $\cdot$ & $\cdot$ & & $\cdot$ & & $\cdot$ & - & & $\cdot$ & & $\cdot$ & & 0 & $0^{\circ}$ & & & & \\
\hline Spongostichomitra elatica (Aliev) & & & & & & & & & & & & & & & & & & & & & $\cdot$ & & & $\bullet$ & & & & & & & & & \\
\hline Staurosphaeretta longispina (Squinabol) & & & & & & & & 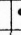 & & & $\cdot$ & & - & & & & & & & & & & & & & & & & & & & & \\
\hline Stichomitra communis Squinabol & & & & & & $\bullet$ & & & & & & & & & & & & & $\cdot$ & & & & $\cdot$ & & & o & & & & & & & \\
\hline Stichomitra mediocris (Tan) & & & & $\bullet$ & $\cdot \bullet$ & $\bullet$ & & & & & & & & & & & & & $\bullet$ & & & & & & & & & & & & & & \\
\hline Thanarla brouweri (Tan) & $\cdot \cdot$ & $\cdot 1$ & $\bullet \bullet$ & $\cdot$ & $\cdot-$ & $\bullet$ & $\cdot$ & - & $\bullet$ & -1 & $\cdot$ & $\cdot$ & $\cdot \bullet$ & & & $\cdot$ & $\cdot$ & $\cdot$ & $\bullet$ & & $\cdot$ & $\cdot$ & $\cdot$ & $\cdot \bullet$ & $\cdot \bullet$ & $\bullet$ & I & $\cdot$ & $\cdot 0$ & 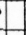 & $\cdot$ & & - \\
\hline Thanarla carboneroensis O'Dogherty & 1 & to & & & & & & & & & & & & & & $\cdot$ & $\bullet$ & 1 & & & & & & & & & & & & & & & \\
\hline Thanarla lacrimula (Foreman) & $\cdot \cdot$ & - 0 & $\circ \cdot$ & $\bullet$ & & & & & & & & & & $\bullet$ & & - & $\cdot \bullet$ & $\cdot$ & - & & & & & & & & - & & $\cdot$ & o & o & & \\
\hline Thanarla pacifica Nakaseko \& Nishimura & $\cdot 0$ & $\cdot 0$ & $\cdot \cdot$ & $\bullet$ & & & & & 0 & & & & & $\cdot$ & & - & $\bullet$ & & - & & & & & & $\cdot$ & & - & & - 0 & & $\circ \cdot$ & $\bullet$ & o \\
\hline Thanarla pseudodecora (Tan) & $\cdot$ & $\cdot$ & & & & & & & 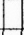 & & & & & & & - & $\bullet \bullet$ & $\cdot$ & & & & & & & & & & & $\bullet$ & & & & \\
\hline Torculum bastetani O'Dogherty & & & & & $\cdot 0$ & $\bullet$ & - & 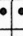 & $\cdot$ & & $\cdot$ & $\cdot$ & $\cdot$ & & - & & & & & & $\cdot$ & & & & $\cdot$ & $\cdot$ & . & & & & & & \\
\hline riactoma echiodes Foreman & & & & $\bullet$ & $\bullet$ & - & $\bullet$ & -10 & & & $\bullet$ & & & & & & - & & & & & & & & & & & & - & & & $\cdot$ & \\
\hline Triactoma hybum Foreman & & $\bullet$ & & $\cdot$ & - 0 & $\bullet$ & - & - 0 & $\cdot$ & & 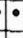 & & & $\bullet$ & & & • & & - & & & & & & & & & & $\cdot$ & & & & \\
\hline Trisyringium capellinii Vinassa & & & & & & & - & 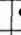 & 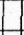 & & 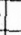 & & & & & & & & & & & & & & & & & & & & & & \\
\hline Turbocapsula costata (Wu) & & & & $\bullet$ & & & $\bullet$ & - & & & $\bullet$ & $\cdot$ & $\bullet$ & & & & & & - & & $\cdot$ & & $\cdot 0$ & & - & $\cdot$ & - & & & & & & \\
\hline Ultranapora praespinifera Pessagno & & & & $\bullet$ & - 0 & & 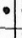 & - 1 & $\bullet$ & & & $\cdot$ & $\cdot \cdot$ & & & & $\cdot$ & 1 & & & $\circ$ & & & & & & & & & & & & \\
\hline Xitus alievi (Foreman) & $\cdot \cdot$ & $\bullet \cdot$ & $\cdot \cdot$ & & & & & & & & & & & $\bullet$ & & 0 & $\bullet \cdot$ & $\cdot$ & & & & & & & & & & & $\cdot$ & $\bullet$ & $\cdot$ & & - \\
\hline Xitus clava (Parona) & $\cdot$ & $\cdot 0$ & $\cdot \bullet$ & & $\cdot \cdot$ & $\bullet$ & $\bullet$ & - & $\bullet$ & $\bullet$ & & - & $\cdot$ & - & $\cdot$ & o & - $\bullet$ & $\cdot$ & $\bullet$ & & $\circ$ & & $\cdot 0$ & & $\bullet \cdot$ & $\cdot$ & & $\cdot$ & $\circ \cdot$ & $\bullet$ & $\cdot \bullet$ & & - \\
\hline Xitus elegans (Squinabol) & & $\bullet$ & $\cdot \cdot$ & & & 0 & - & & - & & 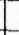 & & & - & & & & & - & & & & - & & & - & & & & & $\cdot$ & & \\
\hline Xitus spicularius (Aliev) & & & & & & & & & & & & & & & & & & & & & & & $\cdot \cdot$ & & & $\bullet$ & & & & & & & \\
\hline Unitary Association (UA) & & & & & & & & & & & & & & & & & & & & & & & & & & & & & & & & & \\
\hline
\end{tabular}

- species identified with certainty; O, species identified with some doubt; Unitary Association numbers 1-9 refer to the biozonation of O'Dogherty (1994) and J34, J35 to that of Jud (1994); 1-3 indicates that the sample ranges from UA1 to UA3, 6/7 indicates that the sample lies between UA6 and UA7; sample series SZ-98-Do-, JA-98-, SZ-98-Q-, JA-00-, SZ-99-Z-, SZ-98-P- and SZ-98-D- are collected from sections Donglha-1, Donglha-2, Qunrang-1, Qunrang-2, Zagapu, Polio and Dazhuqu, respectively. 


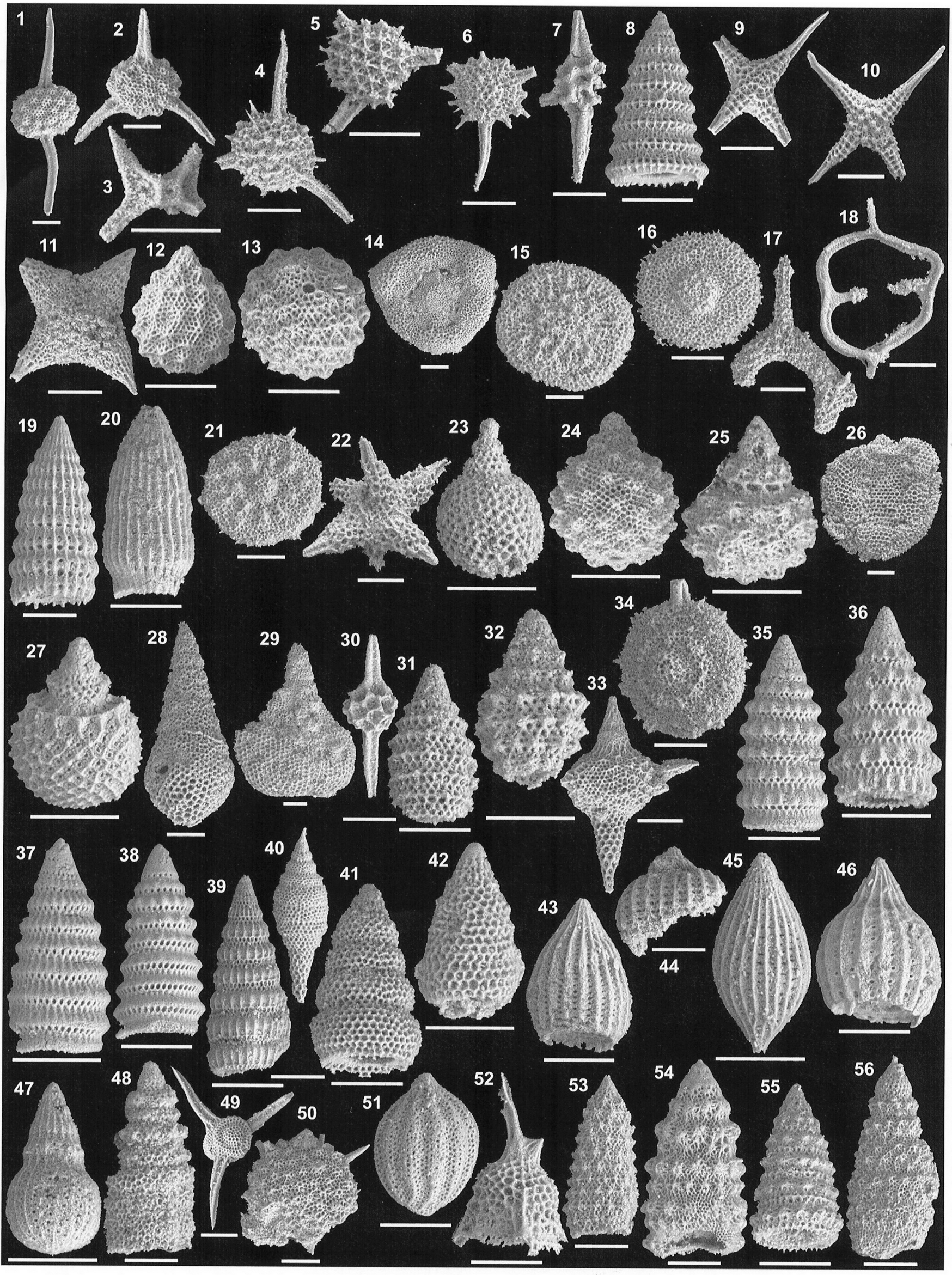


The succession ranges from upper Barremian (chert, sample 19; UAI $H$ asseni Zonc) to upper Aptian (sample 11; UA7-8 T. costata Subzone).

A steeply dipping north-younging sedimentary succession (Qunrang-1, $29^{\circ} 09.303^{\prime} \mathrm{N}, 089^{\circ} 02.702^{\prime} \mathrm{E}$ ) conformably overlies pillow breccias within the ophiolite section on the hillside above the village of Qunrang. Three lithostratigraphic units are recognizable: purplish red bedded $(2-7 \mathrm{~cm})$ chert $(12 \mathrm{~m})$, purplish red siliceous mudstone $(12 \mathrm{~m})$ and thin-bedded $(5-20 \mathrm{~cm})$ fine- to medium-grained volcaniclastic turbidites $(130 \mathrm{~m})$. Chert and siliceous mudstone contain tuffaccous laminae and thin $(0.5-$ $3 \mathrm{~cm}$ ) layers of felsic tuff, sparse at the bottom and abundant at the top (Fig. 2). Sand-sized lithic fragments in the turbidites are mostly of basaltic to andesitic composition. Four matrix-supported conglomerates $(0.2-1.3 \mathrm{~m})$ composed mostly of basalt and less abundant chert clasts sourced from underlying rocks occur in the upper portion of siliceous mudstone. Two clast-supported conglomerates (tens of centimetres) of similar composition lic within turbidites. Outcrop-scale asymmetric folds indicate south-directed thrusting with a sinistral strike-slip component. Rare layer-parallel shear zones traverse the section. To the north, the succession is faulted against a further scetion of ophiolitic basalt. Abundant well-preserved radiolarians indicate that the Qunrang-l section ranges from uppermost Barremian to upper Aptian. Chert samples range from upper Barremian (UA1 H. asseni Zone) to lower Aptian (UA5 H. verbeek Subzone). Overlying siliceous mudstones and volcaniclastic turbidites are upper Aptian (T. costata Subzonc)

The thickest section (Qunrang-2, 29 $08.750^{\prime} \mathrm{N}, 089^{\circ} 01.723^{\prime} \mathrm{F}$ ) of purplish red siliccous mudstone $(40 \mathrm{~m})$ and overlying thinly bedded, fineto medium-grained volcaniclastic turbidites $(310 \mathrm{~m})$ crops out $2 \mathrm{~km} \mathrm{SW}$ of Qunrang-1. It rests conformably on basaltic pillow breccia and dips $55^{\circ} \mathrm{SSW}$. Turbiditic sandstones are lighter in colour and appear more felsic than in the previous section. Some volcaniclastic conglomerates and thick devitrified tuffs occur near the top of the turbidites. The section above the conglomerates is intensely disturbed and folded in the footwal of a north-directed thrust where it is in contact with ultramafic rocks to the south. The entire sequence is assigned to the upper Albian ( $T$. costata Subzone).

A thin sequence (Zagapu, $29^{\circ} 09.113^{\prime} \mathrm{N}, 089^{\circ} 15.419^{\prime} \mathrm{E}$ ) of chert, tuff and siliccous mudstone within a pile of pillow brecia ( $>300 \mathrm{~m}$ thick) conformably overlain by $30 \mathrm{~m}$ of tuff is exposed near Zagapu village along the road from Bainang to Zagapu. Siliceous rocks (4 m thick) occur $40 \mathrm{~m}$ below the tuff and dip $70-85^{\circ} \mathrm{NW}$. Dark grey to purplish red thinly bedded chert $(2.5 \mathrm{~m})$ conformably overlies pillow breccia. Above a shear surface, the chert is overlain by tuff $(1.35 \mathrm{~m})$ covered by thin $(0.2 \mathrm{~m})$ red siliceous mudstone, which is succeeded by pillow breccial. Two radiolarian assemblages recovered indicate that the chert (sample B) is upper Barremian-lower Aptian (UA1 -.5 H. asseni Zone to H. verbeeki Subzone) and the siliceous mudstone (sample D) is upper Aptian (UA7 T. costata Subzone).

Four kilometres south of the road from Lhasa to Xigaze in a creek nea the village of Polio, a steeply south-dipping overturned sequence (Polio, $\left.29^{\circ} 17.096^{\prime} \mathrm{N}, 089^{\circ} 25.286^{\prime} \mathrm{E}\right)$ of cherts $(10.5 \mathrm{~m})$ depositionally ovcrlics pillow breccia. Although minor layer-parallel shearing is present it does not affect the contact. The northern limit of the sequence is tectonically juxtaposed against strongly sheared turbidites of the Xigaze terrane. Black and dark grey chert $(0.9 \mathrm{~m})$ above pillow breceia becomes pale grey up-section where individual chert beds $(2-10 \mathrm{~cm})$ are commonly separated by sepiolitic layers $(3-10 \mathrm{~cm})$, especially abundant between 4 and $9 \mathrm{~m}$ above the basc. Abundant and moderately well-preserved radiolarians indicate that the lower $2.1 \mathrm{~m}$ of chert (samples 1-7) is upper Barremian (UA J34/J35 or $334 / 1$ ).

Four hundred metres upslope from Dazhuqu village, $3 \mathrm{~km}$ solth of the road from Lhasa to Xigaze, a $10-12 \mathrm{~m}$ thick sequence (Dazhuqu. $29^{\circ} 18.828^{\prime} \mathrm{N}, 089^{\circ} 32.058^{\prime} \mathrm{E}$ ) of laminated grecnish grey tulfs and reworked basaltic material $(3-15 \mathrm{~cm})$ depositionally overlies pillow breccia. Minor silicified tuff and mudstone $(2-4 \mathrm{~cm})$ and scarce bluish green chert $(4 \mathrm{~cm})$ occur together with fine-grained reworked basaltio detritus. The section is overturned and dips $65-80^{\circ}$ south. No top to the sequence is exposed but nearby turbidites of the Xigaze terrane to the north are strongly sheared and folded, suggesting at tectonic contact with the Xigaze terrane. A radiolarian assemblage from the base of the sequence is upper Barremian (UA J34/J35 or UA J34/1).

\section{Discussion}

Well-preserved radiolarian faunas, thorough sampling and enhanced resolution of radiolarian biostratigraphy (Jud 1994; O'Dogherty 1994) allow reassessment of the ages of the sedimentary successions overlying the ophiolite and the accurate dating of sedimentary packages within these successions. This places important temporal constraints on the generation of the Dazhuqu terrane ophiolite and provides a basis for detailed sequence correlation (Fig. 4). The oldest deposits are well constrained as upper Barremian in sections at Polio, Dazhucu. Donghla-1, Donghla-2 and Qunrang- 1 . The base of the Qunrang2 section is upper Aptian. This indicates that cruption of ophiolitic basalt occurred before the late Barremian (c. $123 \mathrm{Ma}$ in the Gradstein ef al. (1994) time scale) to the late Aptian (c. $117 \mathrm{Ma}$ ). Although the pillow basalt-chert contact is diachronous throughout the terrane over a $175 \mathrm{~km}$ long zone of outerop it appears that the sediments intercalated with, and immediately overlying, the ophiolitic basalts were deposited within a relatively short (i.c. <10 Ma) interval. With the exception of younger ages for sediments associated with basalts at Qunrang-2 and probably Zagapu, this event, along the length of the terrane, was completed within an even shorter (1-2 Ma) interval.

Investigation of supra-ophiolite deposits in the Yarlung Tsangpo suture zone provides a picture of the patterns of carly sedimentation upon a newly built oceanic floor within an extensional zone that was part of an intra-oceanic subduction system. While the ophiolite was being generated deposition began with accumulation of pillow breccia several hundreds of metres thick. Development of pillow breccia probably occurred along the scarps of normal faults in this extensional setting. A few thin $(5 \mathrm{~cm})$ chert layers were deposited towards the end of pillow breccia accumulation. Background biogenic pelagic sedi-

Fig. 3. Radiolarians from the Dazhuqu terrane (scale bars represent $100 \mu \mathrm{m}$ ). 1, Acceniotyle umbilicata (Rüst); 2, A. diaphorogona Foreman; 3 , Aurisatumalis carinatus (Foreman); 4, Bects gemmattis Wu; 5, B. helente (Schaaf); 6, B. horridus (Squinabol); 7, Cecrops sp. cf. C. septemporatus (Parona); 8, Crolanium puga (Schaaf); 9, Crucella euganea (Squinabol); 10, C. gavalai O'Dogherty; 11, C. hispana O'Dogherty; 12, Cryptamphorella clivosa (Aliev); 13, C. crepida O'Dogherty; 14, Cyclastrum infindibuliforme Rüst; 15, Dactvliodiscus lenticulatus Jud; 16, Dactyliosphaera maxima (Pessagno); 17, Deviatus diamphidius (Forcman); 18, Dicerosaturnalis amissus (Squinabol); 19, Dictyomitra communis (Squinabol); 20, D). exeeflens (Tan); 21, Godia decoma (Li \& Wu); 22, Hexapyramis pantanellii Squinabol; 23, Hiscocapsa asseni (Tan); 24, H. gratterinki (Tan); 25, H. kaminogroensis (Aita); 26, H. orca Foreman; 27, H. uterculus (Parona); 28, Obeliscoites perspiculls (Squinabol); 29, O. vinassai (Squinabol); 30, Pantanellium lancesle (Parona); 31, Parvicingula boesii (Parona); 32, P. usotanensis Tumanda; 33, Podobursa tythopora (Foreman); 34, Pseudoculophacus (?) florealis Jud; 35. Psendodictyomitra carpatica (Lozyniak); 36, P. hornatissima (Squinabol); 37, P. leptoconica (Foreman); 38, P. Iilyae (Tan); 39, P. pentacolcensis Pessagno; 40, Pseudoeucyrtis hanni (Tan); 41, Stichomitra conmunis Squinabol; 42, S. mediocris (Tan); 43, Thanula brouweri (Tan); 44, T. carboneroensis O'Dogherty; 45, T. lacrimula (Foreman); 46, T. pacifica Nakascko \& Nishimura; 47, T. pseudodecora (Tan); 48, Torculum buslerani O'Dogherty; 49, Triactoma hyhum Foreman; 50, Trisyringium capellinii Vinassa; 51, Turbocapsula costata (Wu); 51, Ultramapora praespinifera Pessigno; 53, Xitus alievi (Foreman); 54, X. clava (Parona); 55, X. elegans (Squinabol); 56, X. spicularilus (Aliev). 


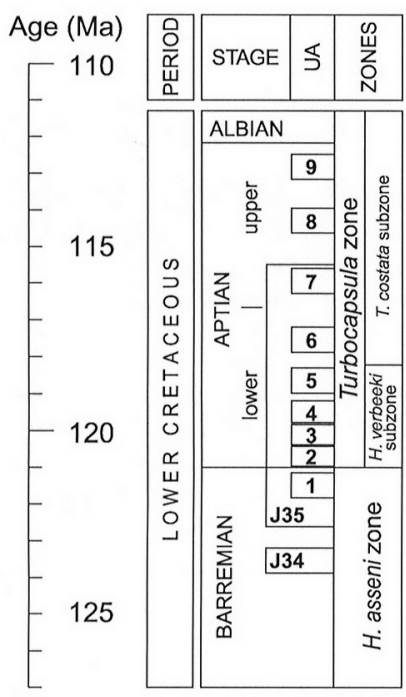

\section{Qunrang-1}
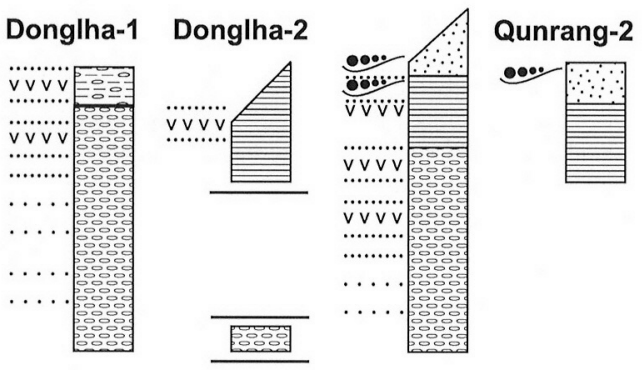

Zagapu
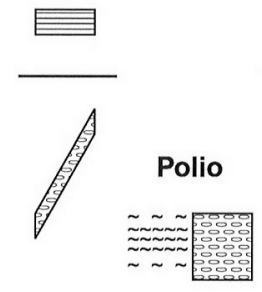

Dazhuqu
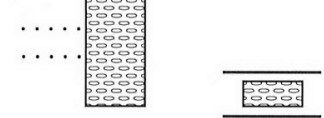

年

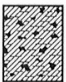

Fig. 4. Correlation chart of the Dazhuqu terrane radiolarian-bearing deposits with lithologies plotted against biostratigraphic (after O’Dogherty 1994; Jud 1994) and chronostratigraphic (after Gradstein et al. 1994) scales. Non-horizontal boundaries indicate uncertainties in age. (For key see Fig. 2.)

mentation was established along the terrane by the late Barremian after the cessation of pillow breccia development. In the easternmost sections, the earliest (late Barremian) accumulation of pelagic chert was swamped by deposition of glassy basaltic detritus that is now locally altered to sepiolite. In the central and western portions of the terrane, chert deposition continued until the late Aptian (Donglha-1) or had terminated by then (other sections). It was interrupted by ash falls from an adjacent volcanic arc and bottom traction currents that reworked tuffaccous materials. Pelagic sedimentation progressed into accumulation of hemipelagic siliceous mudstone in the late Aptian at Donglha-2, Qunrang-1 and Zagapu. At Qunrang-2, sedimentation started with deposition of siliceous mudstones in the late Aptian. The frequency of ash falls and activity of bottom traction currents varied through space and time. Hemipelagic sedimentation at Qunrang- I was interrupted by deposition, from mudflows, of several matrix-supported conglomerates composed of basalt and chert clasts sourced from stratigraphically lower rocks. During the late Aptian, development of pillow breccia resumed at Zagapu, and later switched to thick tuff accumulation. At Qunrang, volcaniclastic turbidite sedimentation took over from hemipelagic sedimentation in the beginning of the late Aptian, when up to $300 \mathrm{~m}$ of finc- 10 medium-grained turbidites were deposited during a short period. The absence of carbonate deposits in the marine sedimentary veneer on the ophiolite indicates sedimentation below the carbonate compensation depth.

Sequences and ages of lithologies in the sedimentary succession vary between sections even where they are only separated by a few kilometres. We interpret such variations as a result of sediment accumulation in small semi-isolated basins on the ophiolitic bascment. This is in accord with the interpretation of the ophiolite belt in the Xigaze area as a tectonic collage of individual massifs genetically related to locally different settings within an overall supra-subduction zone environment (Iébert et al. 2000, 2001). Sections in the western and central parts of the study area exhibit coarsening-upward tendencies, which is especially clear at Qunrang-1. The upper portion of this section (above the pillow breccia) documents a change from pelagic (chert) to hemipelagic (siliceous mudstone) and finally to volcaniclastic turbidite sedimentation. This marked coarsening up-section tendency probably reflects development of a sediment dispersal system associated with a volcanic arc and its progradation onto a zone of newly built supra-subduction zone oceanic floor. The basaltic to andesitic sourcc of volcaniclastic turbidites in the Dazhuqu tcrrane differs from the source of the Xigaze terrane sandstones, which are considerably more felsic (Dürr 1996). Examinations of the sedimentology and radiolarian assemblages in sections overlying pillow basalts of the Dazhuqu terrane ophiolite indicate the rapid development of the ophiolite over a short mid-Cretaceous interval in a Tcthyan intra-oceanic island are setting isolated from any continental landmass such as that inferred to have existed within Tethys in the model of Aitchison et al. (2000).

We thank members of the Tibetan Geological Survey (Team \#2) and Tibetan Geological Society, whose efforts have helped to make this research possible. Many of these friends have assisted with arranging logistics and permission. We thank L. O'Dogherty, R. Jud and J. Guex for their kind permission to use their unpublished data and for fruitful discussions of the nature and applications of Unitary Associations and discrete biostratigraphic scales. This work was supported by grants (to J.C.A.) from the Research Grants Council of the Hong Kong Special Administrative Region, China (Projects HKU7102/98P, 7299/99P and $7069 / 0 \mathrm{lP}$ ). The constructive reviews of T. Danclian and T. Argles helped to improve the manuscript and are gratefully acknowledged.

\section{References}

Abrajivitch, A., AItchison, J.C. \& Al.i, J.R. 2001. Paleomagnetism of the Dazhuqu Terrane, Yarlung Zangbo Suture Zone, Southern Tibet. EOS Transactions. Anerican Geophlysical Union, 82(47), 1314.

AtTchison, J.C., BADENCiZHL, \& DAVIS. A.M. IIT AL. 2000. Remnants of a Cretaceous intra-oceanic subduction system within the Yarlung--Zangbo suture (southern Tibet). Eath and Planetary Sctence Lefters, 183, 231-244.

Atrchison, J.C., Abrajl:vitch, A. \& ALI, J.R. IT AL. 2002a. New insights into the evolution of the Yarlung Tsangpo suture zone, Xizang (Tibet), China. Episodes, 25, 90-94.

Aitchison, J.C., DAvis, A.M., Banfing the India-Asia collision: the Lower Miocene Gangrinboche conglomerates, Yarlung Tsangpo suture zonc, SE Tibet. Joumal of Asian Larth Sciences, 21(3), $253 \quad 265$.

Artchison, J.C., DAvis, A.M. \& Al., J.R. it A.. 2003. Stratigraphic and sedimentological constraints on the age and tectonic cvolution of the Neotethyan ophiolites along the Yarlung Tsangpo suture zone, Tibet. In: Du.k, Y. \& Robinson, P.T. (eds) Ophioliles in Earth /fistory. Geological Society, London, Special Publications, in press. 
Am.jigre, C.I., Courtili.ot, V. \& Tapponniez, P. et at. 1984. Structure and evolution of the Himalaya-Tibet orogenic belt. Nature, $307,17 \cdots 22$.

Baumgartner, P.O., O'Dogherty, L., Gorican, S., Urouhart, E., Pillevvuit, A., Di: Wever, P. (cds) 1995. Middle Jurassic to Lower Cretaceou. Radiolaria of Tethys: Occarrence, Systematics, Biochronology. Mémoires de Gćologie, Université de Lausanne, 23.

Burbau ol: Gliology anid Minirat Rasources or Xizang; Autonomous REGION 1993. Regional Geology of Xizang (Tibet) Autonomous Region. Pcoplc's Republic of China, Ministry of Geology and Mineral Resources Geological Memoirs Series 1, 31

Burg, J.P. 1983. Carte Géologique du Sud du Tibet, scale 1:500,000. CNRS, Paris.

Buro, J.P. \& Chin, G.M. 1984. Tectonics and structural zonation of southem Tibet, China. Nature, 311, 219-223

Davis, A.M., Aitchison, J.C., Badengzhu, Luo, H. \& Zrabriy, S. 2002 Palcogene island are collision-telated conglomerates, Yarlung. Tsangpo suture zone, Tibet. Sedimentary Geology, 150, 247-273.

Dürr, S.B. 1996. Provenance of Xigaze fore-arc basin clastic rocks (Cretaceous, South Tibet). Geological Society of America Bulletin, 108, 609-684.

Einsei.E, G., LiU, B. \& DÜrr, S. IT $M$. 1994. The Xigaze forcarc basin; cvolution and facies archilecture (Cretaceous, Tibet). Sedimentaly Geology, 90, 1--32.

Girardeau, J., Marcoux, J. \& Zao, Y. 1984. Lithologic and tectonic environment of the Xigaze ophiolite (Yarlung Zangbo suture zone, southern Tibet, China), and kinematies of its emplacement. Eclogae Geologicae Helvetiae, 77 $153-170$.

Girardenu, J., Mercier, J.C.C. \& ZAO, Y. 1985a. Structure of the Xigaze Ophiolite, Yarlung Zanglso suture zone, southern Tibet, China; genctic implications. Tectonics, 4, 267-788.

Grrardisu, J., Mrircier, J.C.C. \& \%Ao, Y. 1985b. Origin of the Xigaze Ophiolite, Yarlung Zangbo suturc zone, southern Tibet. Tectonophysics, 119, 407-433.

Göpli, C., Allìgri, C.J. \& Rong, H.X. 1984. Lead isotopic study of the Xigaze ophiolite (Tibet); the problem of the relationship between magmatites (gabbros, dolerites, lavas) and tectonites (harzburgites). Earth and Planetary Science Letters, 69, 301-310.

Graistein, F.M., Agtmerberg, F.P., Ogg, J.G., Hardenbol, J., van Vien, P. Thierry, J. \& HUANG, Z. 1994. A Mesozoic time scale. Joumal of Geophysical Research, 99, B24051-B24074.

Gul:x, J. 1991. Biochronological Comelations. Springer, Berlin.

Héblirt, R., Beaumoin, G., Varkat.yy, V., Huot, F., Wang, C.S. \& liu, Z.F 2000. Yarlung 7angbo ophiolites, southern Tibet revisited, 15th Himalaya Karakorum Tibet workshop, Chengdu. Earh Science troniers, 7, 124-126.

Hébert, R., Wangi, C. \& Varmalvy, V. et a.. 2001. Yarlung Zangbo ophiolites, southern Tibet revisited, 16th Himalaya-Karakorum-Tibet workshop, Graz, Austria I6th HKT abstracts. Jownal of Asian Earh Sciences, 19(3a)), 27-28.

JaDoul, F., Brzkr , F. \& GarzanTt, E. 1998. The Tethys Ilimalayan passive margin from Late Triassic to Early Cretaceous (South Tibet). Jounal of Asian Earth Sciences, 16, 173-194

JUD, R. 1994. Biochronology and Systematics of Early Cretaceous Radiolaria of the Western Tethlys. Mémoires de Gćologic, Université de Lausanne, 19

LI, H.-S. \& W W , H.-R. 1985. Radiolaria from the Cretaccous Congdu Formation in southern Xizang (Tibet). Acta Micropalaeontologica Sinica, 2, 61--76.

Liu, G. \& EINSFi E, G. 1994. Sedimentary history of the Tethyan basin in the Tibetan Himalayas. Geologische Rundschau, 83, 32-61.

LIU, J.B. \& ArTchison, J.C. 2002. Upper Paleocene radiolarians from the Yaundrok mélange, south Xizang (Tibet), China. Micropaleontology, 48(Suppl. 1), $145-154$.

LIU, JB AITCHISON JC BAIJINGZHU, DAVIS, A M JIABRIV S. L LUO, H. \& McDermi1, I. Badengzhu, Davis, A.M., Ziabrev, S.V., Luo, H. \& McDermid, I. 2000. Yamdrok Melange, South Tibet, 15th Himalaya-Karakorum-Tibet workshop, Chengdu. Earth Science Fiontiers, 7, 127

Marcoux, J., DE: Wrvek, P. \& Nicolas, A. ET A. 1982. Preliminary report of depositional sediments on top of volcanic member; Xigaze Ophiolite (Yarlung Zangbo suture zonc); South Xingang (Tibct). Ofioliti, 2/3, 395-396.

MATSUOKA, А., KOBAYASH, K., NAGAHASH, T., YANG, Q., WANG, Y.J., TAKEI M. \& ZiNG, Q.G. 2001. Farly Middle Jurassic (Aalenian) radiolarian fauna from the Xialu chert in the Yarlung Zangbo Suture Zone, southern Tibet. In: Metcalifi, I., Smitu, J.M.B., Morworol, M. \& Davidson, I. (eds) Frnmal and Floral Migrations and Evolution in SE Asia-Australasia. Balkema, Lisse, $105-110$.

Matsuoka, A., Yang, Q., Kobaynshi, K., TAkil, M., Nagahashi, T., Zing, Q.G. \& WANG, Y.J. 2002. Jurassic Cretaceous radiolarian biostratigraphy and sedimentary enviromments of the Ceno-Tethys: records from the Xialu (Chert in the Yarlung-Zangbo Suture Zone, southern Tibet. Jomnd of Asian liath Sciences, 20. 277-287

McDlrmid, I. Atrcilson, J.C. Badengzizh, \& Davis, A.M. 2001 il. The Zedong Terrane: an intra-oceanic magmatic are assemblage Tibet. I goth Himalaya Karakorum-Tibet workshop, (iraz, Austria loth IIKT abstracts, Lotrnal of Asian Earth Sciences, 19(3a), 44.

McDermid, I., Attchison, J.C., Davis, A.M., Harrison, T.M. \& Grovi, M 2001b. The Zedong Terrane: a Jurassic intra-oceanic magmatic are within the Yartung Zangbo Suture Zone of Southeastern Tibet f61h flimalilya Karakorum-Tibet workshop, Graz, Nustria loth HKT abstracts. foumal of Asian Earth Sciences, 19(3a), 44-45.

McDirmid, I., Altrehison, J.C., DAvis, A.M. Harrison, T.M. \& Grovi: M. 20012 The Zedong terrane: a Late Jurassic intra-oceanic magmatic alle within the Yarlung Zangbo suture zone, southeastem Tibet. Chemical (ioologl, 187. $267-277$.

Nicolas, A., Grkardiau, J. \& Marcoux, J. Mt M. 1981. The Xigare ophiolite (Tiber); a peculiar oceanic lithosphere. Nuture, 294, 414417.

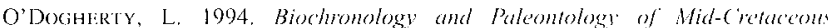
Radiolarians from Northern Apennines (haly) and Befic (ordillera (Spain). Mémoires de Géologie, Université de Latusanne, 21

Pissagino, E.A. 1976. Radiolarian zonation and stratigraphy of the Upque Cretaceous portion of the Great Valley Sequence, California ('oast Ranges. Micropaleontology: Special Publication, 2, 195.

Proust, F BURG, JP MATF, P TAPONNIER P L T L G \& C14: G 1984. Succession des phases de plissement sur une transversale du tïbet

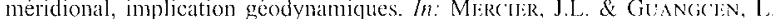

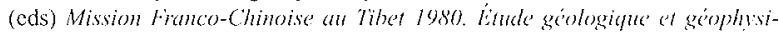

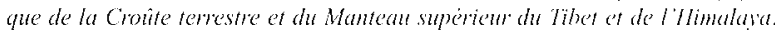
CNRS, Paris, $385 \cdot 392$

Rntschbacher, L., Frisch, W. \& Liu, G. 1994. Distributed defomation in southern and western Tibet during and after the India- Asta collision. .omwat of Geophwical Research, 99, 19917-19945.

Sentli, M.P., Winidiliy. B.F. \& Cowarid. M.P. i.T M.. 1987. The closing of Tethys and the tectonics of the Himalaya. Geological Soriets of Americe Bulletin, 98,678.701.

SHACkI.FTON, R.M. 1981. Structure of southern Tibet; repont on a traverse from Lhasa to Khatmandu organised by Academia Sinica. Jemmal of Simconret Geology, 3,97 105

WAN, X., Wang, L., WANG, C. \& JANSA, L. 1998. Discovery and significance of Cretaceous fossils from the Xigaze foreare basin, Tiber. Jounal of tsian Earth Sciences, $16,217-223$

Wang, X.B., BAO, P.S., DFNG. W.M. \& Wang, F.G. (eds) 1087. Tertomic Evolution of the Lithosphere of the Rlimalasas: Xizang (Tithet Ophiolite People's Republic of China Ministry of (jeology and Mineral Resources Geological Memoirs Series 3, 8

Wirdmann, J. \& Dürr, S.B. 1995. First ammonites from the Mid- to Upper Cretaceous Xigaze Group, South Tibet, and their significance. Newrelters on Stratigraphi, 32, 17-26

WU, H.R. 1986. Some new genera and speeies of Cenomanian Radiolaria from southern Xizang (Tibet). Aca Micropalaeomologica Simica, 3, 347 360

WU, H.R. 1993. Upper Jurassic and Lower Cretaceous radjolarians of Xialu chert, Yarlung Zangbo ophiolite belt, southern Tibet. In: Buntroms, J.R. \& Murcinsy, B.L. (eds) Radiolaria of Giant and Subgiant litelds of Asia. Nazarov Memorial Volume. Micropaleontology Special Paper, 6, 115 136.

YIN, A. \& HARrison, T.M. 2000. Geologic Evolution of the Jimilayan. 'Tibetum Orogen. Anmal Review of Earth and Planetary Sciences, 28, 211280.

Yin, A., Harrison, T.M., Ryerson, F.J., Chin, W.J., Kind, W.S.F. \& Colei.ani), P. 1994. Tertiary structural evolution of the Cangdese thrust system in southeastern Tibet. Joumal of Geophysical Researh, 99, 1817518201

Yin, A., Harrison, T.M. \& Murphr, M.A. IT M. I999. Tertiary delomation history of southeastern and southwestern Tibet during the Indo- $\Lambda$ sian collision. Geological Society of America Bullotim, 111, 16441664.

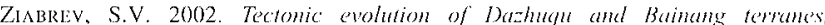
Yathang-..Zangbo sutwe, Tibet as constrained by madiolatan biostratignass: PhD thesis, University of I long Kong.

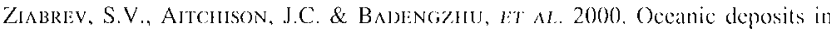
the Yarlung-Tsangpo suture zone: structural setting. radiolarian ages and their tectonic implications, 15th llimalaya Karakorum Tibet workshop, Chengdu 15th HKT abstracts. Farth Science Frontiess, 7, 118 